\title{
Influence of synthesis method on structural and magnetic properties of cobalt ferrite nanoparticles
}

\author{
Sašo Gyergyek • Darko Makovec • Alojz Kodre • \\ Iztok Arčon · Marko Jagodič • Miha Drofenik
}

Received: 14 November 2008/ Accepted: 16 December 2009

(C) Springer Science+Business Media B.V. 2009

\begin{abstract}
The Co-ferrite nanoparticles having a relatively uniform size distribution around $8 \mathrm{~nm}$ were synthesized by three different methods. A simple coprecipitation from aqueous solutions and a coprecipitation in an environment of microemulsions are low temperature methods $\left(50^{\circ} \mathrm{C}\right)$, whereas a thermal decomposition of organo-metallic complexes was performed at elevated temperature of $290{ }^{\circ} \mathrm{C}$. The X-ray diffractometry (XRD) showed spinel structure, and the high-resolution transmission elec-
\end{abstract}

Electronic supplementary material The online version of this article (doi:10.1007/s11051-009-9833-5) contains supplementary material, which is available to authorized users.

S. Gyergyek ( $\square) \cdot$ D. Makovec $\cdot$ A. Kodre .

I. Arčon - M. Drofenik

"Jozef Stefan" Institute, Ljubljana, Slovenia

e-mail: saso.gyergyek@ijs.si

\section{A. Kodre}

Faculty of Mathematics and Physics, University of Ljubljana, Ljubljana, Slovenia

\section{Arčon}

University of Nova Gorica, Nova Gorica, Slovenia

\section{Jagodič}

Institute of Mathematics, Physics and Mechanics,

Ljubljana, Slovenia

M. Drofenik

Faculty of Chemistry and Chemical Engineering,

University of Maribor, Maribor, Slovenia tron microscopy (HRTEM) a good crystallinity of all the nanoparticles. Energy-dispersive X-ray spectroscopy (EDS) showed the composition close to stoichiometric $\left(\sim \mathrm{CoFe}_{2} \mathrm{O}_{4}\right)$ for both co-precipitated nanoparticles, whereas the nanoparticles prepared by the thermal decomposition were Co-deficient $\left(\sim \mathrm{Co}_{0.6} \mathrm{Fe}_{2.4} \mathrm{O}_{4}\right)$. The X-ray absorption near-edge structure (XANES) analysis showed Co valence of $2+$ in all the samples, Fe valence $3+$ in both coprecipitated samples, but average Fe valence of $2.7+$ in the sample synthesized by thermal decomposition. The variations in cation distribution within the spinel lattice were observed by structural refinement of Xray absorption fine structure (EXAFS). Like the bulk $\mathrm{CoFe}_{2} \mathrm{O}_{4}$, the nanoparticles synthesized at elevated temperature using thermal decomposition displayed inverse spinel structure with the Co ions occupying predominantly octahedral lattice sites, whereas coprecipitated samples showed considerable proportion of cobalt ions occupying tetrahedral sites (nearly $1 / 3$ for the nanoparticles synthesized by co-precipitation from aqueous solutions and almost $1 / 4$ for the nanoparticles synthesized in microemulsions). Magnetic measurements performed at room temperature and at $10 \mathrm{~K}$ were in good agreement with the nanoparticles' composition and the cation distribution in their structure. The presented study clearly shows that the distribution of the cations within the spinel lattice of the ferrite nanoparticles, and consequently their magnetic properties are strongly affected by the synthesis method used. 
Keywords Nanoparticles - Spinel ferrite ·

Structure $\cdot$ Synthesis $\cdot$ EXAFS

\section{Introduction}

Magnetic nanoparticles with uniform size distributions are of great interest because of their unique magnetic properties, dominated by superparamagnetism (Kodama 1999; Batlle and Labarta 2002). Stable suspensions of the superparamagnetic nanoparticles, so-called magnetic fluids (or ferrofluids) are important in different technological applications (Rosenweig 1932). Magnetic nanoparticles can also be used in biomedical applications (Pankhurst et al. 2003; Elster and Burdette 2001; Häfeli et al. 1997; Widder et al. 1978; Senyei et al. 1978; Mosbach and Schröder 1979; Arruebo et al. 2007; Gilchrist et al. 1957; Veverka et al. 2007). They are already used in diagnostics for enhancement of contrast during NMR imaging (Elster and Burdette 2001) and in detection of biomolecules by magnetic separation (Häfeli et al. 1997). Numerous new applications are developed also for therapeutics, for example in targeted drug delivery (Widder et al. 1978; Mosbach and Schröder 1979; Arruebo et al. 2007) or in magnetic hyperthermia (Gilchrist et al. 1957; Veverka et al. 2007).

Among different magnetic materials, cobalt ferrite nanoparticles have a special place because of their high magnetocrystalline anisotropy and large magneto-optical coefficients (Smit and Wijn 1959; Fontjin et al. 1999). Cobalt ferrite is a member of ferrites with a spinel structure. In the spinel structure with general formula $\mathrm{AB}_{2} \mathrm{O}_{4}$, oxygen atoms form cubic close packing where $\mathrm{A}$ and $\mathrm{B}$ cations occupy tetrahedral and octahedral interstitial lattice sites. Generally, the spinel ferrite structure can be represented by $\left(\mathrm{Me}_{1-}^{2+} \mathrm{Fe}_{X}^{3+}\right)\left[\mathrm{Me}_{X}^{2+} \mathrm{Fe}_{2-}^{3+}\right] \mathrm{O}_{4}$ formula, where round and square brackets represent tetrahedral and octahedral sites, respectively. The degree of inversion $X$ is the proportion of the trivalent cation $\left(\mathrm{Fe}^{3+}\right)$ occupying tetrahedral sites (Smit and Wijn 1959).

Magnetic properties of ferrites are directly related to the distribution of the cations over tetrahedral and octahedral lattice sites. Since the magnetic moments of the ions are ordered parallel within each sublattice and antiparallel between both sublattices, the difference in the magnetic moments between both sublattices gives magnetic moment to the ferrite crystal (Smit and Wijn 1959).
Bulk cobalt ferrite is presumed to display the inverse spinel structure $(X=1)$, with all $\mathrm{Co}^{2+}$ ions exclusively at octahedral lattice sites (Smit and Wijn 1959). In the case of nanoparticles, the structure can substantially deviate from the bulk. The flexibility of the crystal structure is particularly pronounced in the spinel ferrite nanoparticles (Sato et al. 1990; Kamiyama et al. 1992; Jeyadevan et al. 1994; Hamdeh et al. 1997; Ammar et al. 2004; Makovec and Drofenik 2008; Carpenter et al. 1999; Calvin et al. 2002; Makovec et al. 2009; Morrison et al. 2004; Li et al. 2000; Sivakumar et al. 2007; Tirosh et al. 2006). For example, $\mathrm{ZnFe}_{2} \mathrm{O}_{4}$ shows paramagnetic behavior as bulk material due to its normal spinel structure, with $\mathrm{Zn}$ ions incorporated almost exclusively at tetrahedral sites. When $\mathrm{ZnFe}_{2} \mathrm{O}_{4}$ is prepared in the form of nanoparticles, it becomes ferrimagnetic due to a partial migration of $\mathrm{Zn}$ to the octahedral sites (Sato et al. 1990; Kamiyama et al. 1992; Jeyadevan et al. 1994; Hamdeh et al. 1997; Ammar et al. 2004; Makovec and Drofenik 2008). The degree of inversion $X$ for $\mathrm{ZnFe}_{2} \mathrm{O}_{4}$ nanoparticles prepared by coprecipitation was determined to increase with decreasing particle size (Kamiyama et al. 1992). Besides on the size, the degree of inversion also depends on the synthesis method (Ammar et al. 2004; Tirosh et al. 2006).

It seems that the synthesis method also has a marked impact on the cation distribution inside the spinel lattice of $\mathrm{CoFe}_{2} \mathrm{O}_{4}$ nanoparticles. Sivakumar et al. (2007) qualitatively showed using Mossbauer spectroscopy and EXAFS that a substantial fraction of $\mathrm{Co}^{2+}$ ions adopts tetrahedral sites in $8 \mathrm{~nm}$-sized $\mathrm{CoFe}_{2} \mathrm{O}_{4}$ nanoparticles prepared using aqueous coprecipitation. Tirosh et al. (2006) compared properties of Co-ferrite nanoparticles with comparable sizes around $10 \mathrm{~nm}$ prepared using different methods. Magneto-optical spectroscopy suggested substantial differences in the incorporation of $\mathrm{Co}^{2+}$ ions within the spinel structure. Based on EXAFS analysis $\mathrm{Li}$ et al. (2000) found Co predominantly at octahedral positions of the spinel structure of Co-ferrite nanoparticles (10-15 nm in size) synthesized using microemulsion method.

In this study, we compare structural and magnetic properties of Co-ferrite nanoparticles of comparable sizes around $8 \mathrm{~nm}$ synthesized using three different methods: an aqueous co-precipitation, a microemulsion method, and a thermal decomposition of oleates. 
In general, the spinel ferrite nanoparticles can be easily synthesized already at low temperatures, for example by using simple co-precipitation of metal ions including $\mathrm{Fe}^{2+}$. Subsequent oxidation of Fe(II) hydroxide results in direct formation of the spinel ferrite:

$$
\begin{aligned}
& \mathrm{Co}^{2+}+2 \mathrm{Fe}^{2+}+6 \mathrm{OH}^{-}+1 / 2 \mathrm{O}_{2} \\
& \quad \rightarrow \mathrm{CoFe}_{2} \mathrm{O}_{4}+3 \mathrm{H}_{2} \mathrm{O}
\end{aligned}
$$

The co-precipitation from aqueous solutions is a relatively simple method and is therefore suitable for mass production; however, it provides only a limited control over particle size and the size distribution. In general, particle size is mainly determined by the relative rates of two processes: the nucleation and the growth, both primarily dependent on the level of supersaturation. The simultaneous occurrence of both processes results in a relatively broad size distribution. In practice, it is also difficult to maintain a homogeneous supersaturation level throughout the whole volume of the reaction vessel during coprecipitation (Sugimoto 2001).

A possible strategy for controlling particle size is the co-precipitation inside the confined space of reverse micelles - tiny droplets of aqueous phase that are embedded with a surfactant in an oil phase of a microemulsion. In that case, the particle size is decisively determined by the size of the micelles, which is thermodynamically defined, in particular with the water-to-surfactant ratio. The uniform size of the micelles enables synthesis of nanoparticles with a narrow size distribution, and the size can be simply controlled just by changing microemulsion composition, the temperature, or the concentration of reactants dissolved in the aqueous phase of the microemulsion (Pileni 1993; Makovec et al. 2004).

Another possible strategy for synthesis of monodispersed nanoparticles is the use of the method that allows complete separation of the process of nucleation from the process of particle growth (Sugimoto 2001). One of the very few such methods is based on thermal decomposition of metal-organic complexes. During heating of Co- and $\mathrm{Fe}$-oleates in a high-boilingpoint liquid medium, the nucleation starts with partial decomposition of oleates at temperatures around $220{ }^{\circ} \mathrm{C}$. At much higher temperature above $280{ }^{\circ} \mathrm{C}$ the nuclei start to grow simultaneously leading to the formation of nanoparticles with extremely narrow size distribution (Park et al. 2004; Sun et al. 2004).

\section{Experimental section}

Materials

Cobalt (II) sulfate heptahydrate $\left(\mathrm{CoSO}_{4} \cdot 7 \mathrm{H}_{2} \mathrm{O}\right.$, Alfa Aesar, 98\%), iron (II) sulfate heptahydrate $\left(\mathrm{FeSO}_{4}\right.$. $7 \mathrm{H}_{2} \mathrm{O}$, Alfa Aesar 99+\%), iron (III) 2,4-pentadionate $\left(\mathrm{Fe}(\mathrm{acac})_{3}\right.$, Alfa Aesar), cobalt 2,4-pentadionate (Co(acac) $)_{2}$ Alfa Aesar), sulfuric acid $\left(\mathrm{H}_{2} \mathrm{SO}_{4}\right.$, Carlo Erba, 96\%), tetramethyl ammonium hydroxide (TMAH, Alfa Aesar, 25\%), $n$-hexanol (Alfa Aesar, 99\%), $n$-hexane (Alfa Aesar, 99\%), methanol (Merck, p.a.), benzyl ether (Alfa Aesar, 98\%), oleic acid (OA, Alfa Aesar, practical 90-95\%), and $n$-hexadecil trimethylammonium bromide (CTAB, Alfa Aesar, 98\%) were used as received, without further purification.

Co-precipitation from aqueous solutions (sample CP)

The nanoparticles CP were synthesized using simple co-precipitation from aqueous solutions. A fresh solution of metal ions $\left(0.05 \mathrm{~mol} / \mathrm{L} \mathrm{Co}^{2+}, 0.10 \mathrm{~mol} / \mathrm{L}\right.$ $\mathrm{Fe}^{2+}$ ) was prepared using $\mathrm{CoSO}_{4}$ and $\mathrm{FeSO}_{4}$. In order to prevent the oxidation of $\mathrm{Fe}^{2+}$ prior to the spinelformation reaction, the $\mathrm{pH}$ value of the solution was set below 1.5 using $\mathrm{H}_{2} \mathrm{SO}_{4}$. $100 \mathrm{~mL}$ of the solution of metal ions (the solution $\mathrm{A}$ ) were added drop-wise to $500 \mathrm{~mL}$ of the solution of the precipitation agent tetramethyl ammonium hydroxide (TMAH) (solution B), with $\mathrm{pH}$ of 12.6. During addition of the solution A the $\mathrm{pH}$ value was maintained constant by simultaneous addition of concentrated TMAH. After the solution A was added, the mixture was aged for $1 \mathrm{~h}$ to allow complete oxidation of $\mathrm{Fe}^{2+}$ and the formation of the spinel ferrite. During the entire process, the mixture was intensively stirred while the temperature was maintained at $50{ }^{\circ} \mathrm{C}$. Finally, the nanoparticles were separated by centrifugation, washed with distilled water and dried at $70{ }^{\circ} \mathrm{C}$ in ambient air.

Co-precipitation in reversed microemulsion (sample ME)

The nanoparticles ME were synthesized in the microemulsion system consisting of $22 \mathrm{wt} \%$ of aqueous phase, $45 \mathrm{wt} \%$ of $n$-hexanol as the oil phase, and $33 \mathrm{wt} \% n$-hexadecil trimethylammonium bromide $(\mathrm{CTAB})$ as the surfactant. The co-precipitation occurred 
when the microemulsion $\mu \mathrm{EA}$ containing the aqueous solution of metal ions $\left(0.05 \mathrm{~mol} / \mathrm{L} \mathrm{Co}^{2+}, 0.10 \mathrm{~mol} / \mathrm{L}\right.$ $\mathrm{Fe}^{2+}$, prepared as for synthesis of the nanoparticles $\mathrm{CP}$ ) was admixed into the equal amount of the microemulsion $\mu \mathrm{EB}$ containing $0.75 \mathrm{~mol} / \mathrm{L}$ of TMAH. During synthesis the temperature was maintained at $50{ }^{\circ} \mathrm{C}$. After an aging time of $1 \mathrm{~h}$ the precipitated nanoparticles were washed with ethanol and with distilled water, and dried in ambient air at $70{ }^{\circ} \mathrm{C}$.

\section{Thermal decomposition (sample TD)}

The hydrophobic, OA-coated nanoparticles TD were synthesized using modified process by Sun et al. (2004) $\mathrm{Fe}(\mathrm{acac})_{3}$ (4 mmol), $\mathrm{Co}(\mathrm{acac})_{2}$, OA (7.3 g) and benzyl ether $(40 \mathrm{~mL})$ were mixed and magnetically stirred under a flow of argon. The mixture was slowly $\left(2.5 \mathrm{~K} / \mathrm{min}\right.$ ) heated to $200{ }^{\circ} \mathrm{C}$ and kept for $2 \mathrm{~h}$ to promote nucleation. After the nucleation period, the mixture was slowly $(2.5 \mathrm{~K} / \mathrm{min})$ heated to reflux temperature $\left(\approx 290{ }^{\circ} \mathrm{C}\right)$ and kept for $1 \mathrm{~h}$. The black colored mixture was cooled to room temperature by removing the heat source. Under ambient conditions, a large amount of methanol was added, and black material was precipitated and separated via centrifugation. Washing with methanol and centrifugation (5,000 rpm, $10 \mathrm{~min}$ ) was used at least three times to thoroughly remove the solvent and impurities. In the end the product was dispersed in hexane and centrifuged (5,000 rpm, $10 \mathrm{~min}$ ) to remove any undispersed material. For XRD characterization, magnetometry and EXAFS the nanoparticles were precipitated from the stable hexane suspension via addition of acetone and centrifugation (5,000 rpm, $10 \mathrm{~min}$ ).

Synthesis of the standard (sample BS)

The structure of as-synthesized nanoparticles was compared to the structure of the nanoparticles after high-temperature annealing (sample BS). The coprecipitated nanoparticles $\mathrm{CP}$ were annealed at $600{ }^{\circ} \mathrm{C}$ for $15 \mathrm{~h}$ in an ambient air. After heating, the sample was quenched by a fast removal from the hot furnace.

\section{Analytical methods}

Phase purity of the nanoparticles was checked using X-ray powder diffractometry (XRD) (PANanalytical $X^{\prime}$ Pert PRO). The particle size, crystallinity, and composition of the nanoparticles were characterized by high-resolution transmission electron microscopy (HREM) coupled with energy-dispersive X-ray spectroscopy (EDX). A field-emission electron-source TEM (JEOL $2010 \mathrm{~F}$ ) was operated at $200 \mathrm{kV}$. For the HREM investigations the nanoparticles were deposited on a copper-grid-supported perforated transparent carbon foil. Quantitative analyses were performed using an EDS microanalysis system (LINK ISIS EDS 300) and Oxford ISIS software. A large number of spectra $(>15)$ was collected from the individual nanoparticles and smaller groups of the nanoparticles. For the quantification of the spectra the $\mathrm{CoFe}_{2} \mathrm{O}_{4}$ ceramic was used as a standard. For each measurement the $\mathrm{Co} / \mathrm{Fe}$ atomic ratio was calculated and the standard deviation of the measurements was determined. The relative standard deviation of the method calculated from 20 measurements of the $\mathrm{Co} / \mathrm{Fe}$ ratio on the standard was found to be inside $\pm 2.6 \%$ $\left(\mathrm{Co} / \mathrm{Fe}_{\text {standard }}=0.502 \pm 0.013\right)$.

In addition to the direct observation by the TEM, the particle size of the product was estimated from the X-ray diffractograms using the Debye-Scherrer method (Cullity 1987) with the crystallographic program Topas2R 2000 (Bruker AXS).

The room-temperature magnetic properties of the nanoparticles were measured with a vibrating-sample magnetometer (VSM) (Lake Shore 7307 VSM). The $10 \mathrm{~K}$ magnetization curves and the temperature dependence of the magnetization, measured under zero-field-cooling (ZFC) conditions and field-cooling (FC) $(H=100 \mathrm{Oe})$ conditions in the temperature range between 2 and $300 \mathrm{~K}$, were measured using a SQUID (Quantum Design SQUID). The magnetization values are given normalized per gram of pure magnetic phase. The mass fraction of the pure magnetic phase in the samples was measured using thermogravimetry.

The local structure in the neighborhood of the constituent metal cations in the nanoparticles was studied by X-ray absorption spectroscopy (EXAFS) and compared to the structure of a "bulk" standard BS, prepared by annealing the nanoparticles $\mathrm{CP}$ at $600{ }^{\circ} \mathrm{C}$. During annealing, the nanoparticles retained their single-phase spinel structure while they coarsened to reach particle size over $60 \mathrm{~nm}$. It is known that the structure of nanoparticles relaxes toward the structure of the bulk at such high temperatures (Makovec and Drofenik 2008; Makovec et al. 2009). For EXAFS, the Co-ferrite samples were 
intimately mixed with $\mathrm{BN}$ and pressed into thin pellets with a total absorption thickness of about 2 above the $\mathrm{Fe}$ and $\mathrm{Co} \mathrm{K}$-absorption edge (7,112 and $7,709 \mathrm{eV}$, respectively). EXAFS spectra at the $\mathrm{K}$ edges of both metallic elements were measured at the C beamline of HASYLAB. A Si (111) double crystal monochromator with resolution of about $1 \mathrm{eV}$ at $7 \mathrm{keV}$ and a dynamic higher-harmonics rejection with the feedback control of the monochromator crystals was used. The intensity of the monochromatic X-ray beam was measured by three consecutive ionization detectors of which the first was filled with a mixture of nitrogen at 650 mbar and Ar at 30 mbar, the second with 380 mbar of $\mathrm{Ar}$, and the third 580 mbar of Ar. The measured samples were placed between the first two detectors. The exact energy calibration with a precision of $0.1 \mathrm{eV}$ or better was established with a simultaneous absorption measurement on the corresponding ( $\mathrm{Fe}, \mathrm{Co}$ ) metal foil placed between the second and the third ionization detector.

The K edge profiles of the constituent metals were scanned with a step of $0.25 \mathrm{eV}$ to enable a precise determination of the edge shape and position. For the EXAFS region equidistant $\mathrm{k}$-steps $\left(\Delta \mathrm{k} \approx 0.03 \AA^{-1}\right)$ were adopted over the joint interval of both edges and to 1,000 $\mathrm{eV}$ above Co K edge, with the integration time of $2 \mathrm{~s} / \mathrm{step}$. Two repetitions were collected for each sample and superimposed to improve the signal-tonoise ratio. The spectra were analyzed with the IFEFFIT code (Ravel and Newville 2005). The FEFF model of the EXAFS signal at the two metal K edges was built from the crystallographic data on the mixed ferrite (Ravel and Newville 2005). Following the argumentation in the study of Calvin (Calvin et al. 2002), a comprehensive FEFF calculation was adopted with a simultaneous relaxation of the two metal spectra, exploiting connections and restraints between the parameters of the EXAFS models of the constituent elements. The advantage of this approach with regard to the separate fitting of individual EXAFS signals lies in the larger phase space almost doubling the number of independent data points and consequently increasing the statistical reliability of determined parameters.

\section{Results and discussion}

The XRD patterns of the synthesized nanoparticles (Fig. 1) show only broad peaks, characteristic for the spinel structure. The average size determined from XRD peak broadening (Table 1) was found to be close to $8 \mathrm{~nm}$ for all of the synthesized nanoparticles and over $60 \mathrm{~nm}$ for the annealed sample BS.

The nanoparticles prepared by co-precipitation from aqueous solutions (sample CP) and in microemulsion (sample ME) were strongly agglomerated (Fig. 2a, b). The agglomeration is expected, because there is no surfactant attached to the surface of the nanoparticles to maintain them in a dispersed state. The nanoparticles prepared by thermal decomposition (sample TD) are, on the contrary, well separated (Fig. 2c), since oleic acid bonded to the surface provides steric repulsion.

In general the nanoparticles had globular shape, only some of the nanoparticles from the sample CP showed elongated shape. The size of the agglomerated nanoparticles was difficult to measure with high precision. For these nanoparticles a rough estimate was made from dark-field images (given in Supplement 1), where individual nanoparticles are clearly visible at the thin edges of the agglomerates (approximately 60 nanoparticles were measured). As expected, the nanoparticles ME synthesized by co-precipitation in microemulsion had narrower size distribution (relative dispersion around 30\%) than the nanoparticles CP (relative dispersion around 60\%), which were synthesized by simple co-precipitation from aqueous solutions. The size distribution was the narrowest in the case of the nanoparticles TD synthesized by thermal decomposition (relative

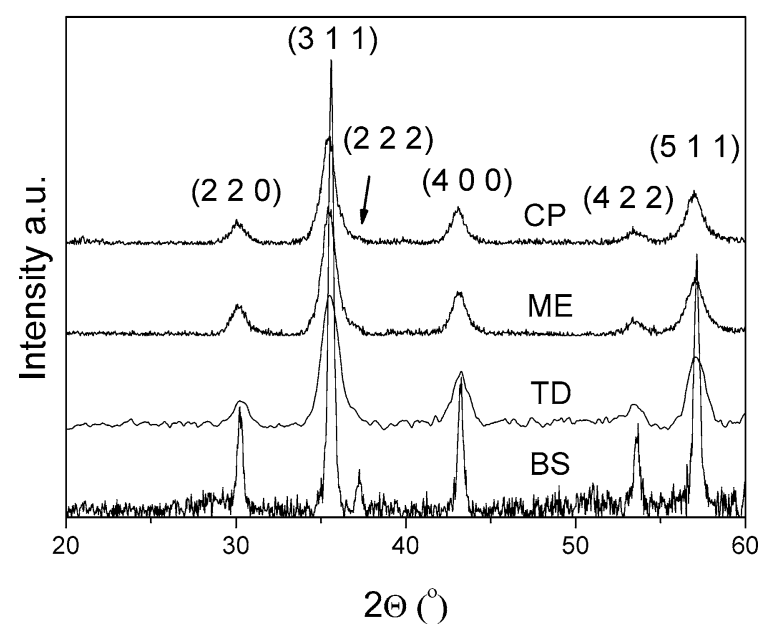

Fig. 1 XRD patterns of the Co-ferrite nanoparticles TD, ME, and $\mathrm{CP}$ and standard $\mathrm{BS}$ 
Table 1 Structural and magnetic properties of the synthesized nanoparticles

\begin{tabular}{|c|c|c|c|c|c|c|c|c|c|c|}
\hline & \multirow{2}{*}{$\begin{array}{l}d_{\mathrm{XRD}} \\
(\mathrm{nm})\end{array}$} & \multirow{2}{*}{$\begin{array}{l}d_{\text {TEM }} \\
(\mathrm{nm})\end{array}$} & \multicolumn{3}{|c|}{ Composition measured using EDS } & \multirow[t]{2}{*}{$X^{\mathrm{a}}$} & \multicolumn{4}{|c|}{ Magnetic properties ${ }^{b}$} \\
\hline & & & Co (at \%) & $\mathrm{Fe}(\mathrm{at} \%)$ & $\mathrm{Co} / \mathrm{Fe}$ & & $\begin{array}{l}M_{10 \mathrm{kOe}}^{\mathrm{c}} \\
(\mathrm{emu} / \mathrm{g})\end{array}$ & $\begin{array}{l}M_{\mathrm{s}}^{\mathrm{d}} \\
(\mathrm{emu} / \mathrm{g})\end{array}$ & $M_{\mathrm{r}} / M_{\mathrm{s}}^{\mathrm{e}}$ & $H_{\mathrm{C}}^{\mathrm{f}}(\mathrm{Oe})$ \\
\hline $\mathrm{CP}$ & 8.7 & $8.2 \pm 5.0$ & $12.62 \pm 0.30$ & $29.91 \pm 0.24$ & $0.422 \pm 0.013$ & 0.79 & 38 & 57 & 0.63 & 800 \\
\hline ME & 7.6 & $10.0 \pm 2.9$ & $14.27 \pm 0.47$ & $28.58 \pm 0.38$ & $0.500 \pm 0.018$ & 0.74 & 44 & 86 & 0.53 & 700 \\
\hline $\mathrm{TD}$ & 8.2 & $9.1 \pm 1.0$ & $8.35 \pm 0.13$ & $33.32 \pm 0.11$ & $0.250 \pm 0.005$ & 0.926 & 46 & 61 & 0.76 & 1,900 \\
\hline BS & 60 & - & - & - & - & 0.931 & 64 & 82 & 0.84 & 1,400 \\
\hline
\end{tabular}

\footnotetext{
${ }^{a}$ Degree of inversion from EXAFS analysis

b The magnetization values were normalized per gram of pure magnetic phase

c Room-temperature magnetization at maximum magnetic field of $10 \mathrm{kOe}$

d Saturation magnetization measured at $10 \mathrm{~K}$

e Remanence-to-saturation magnetization ratio at $10 \mathrm{~K}$

${ }^{\mathrm{f}}$ Coercivity at $10 \mathrm{~K}$
}
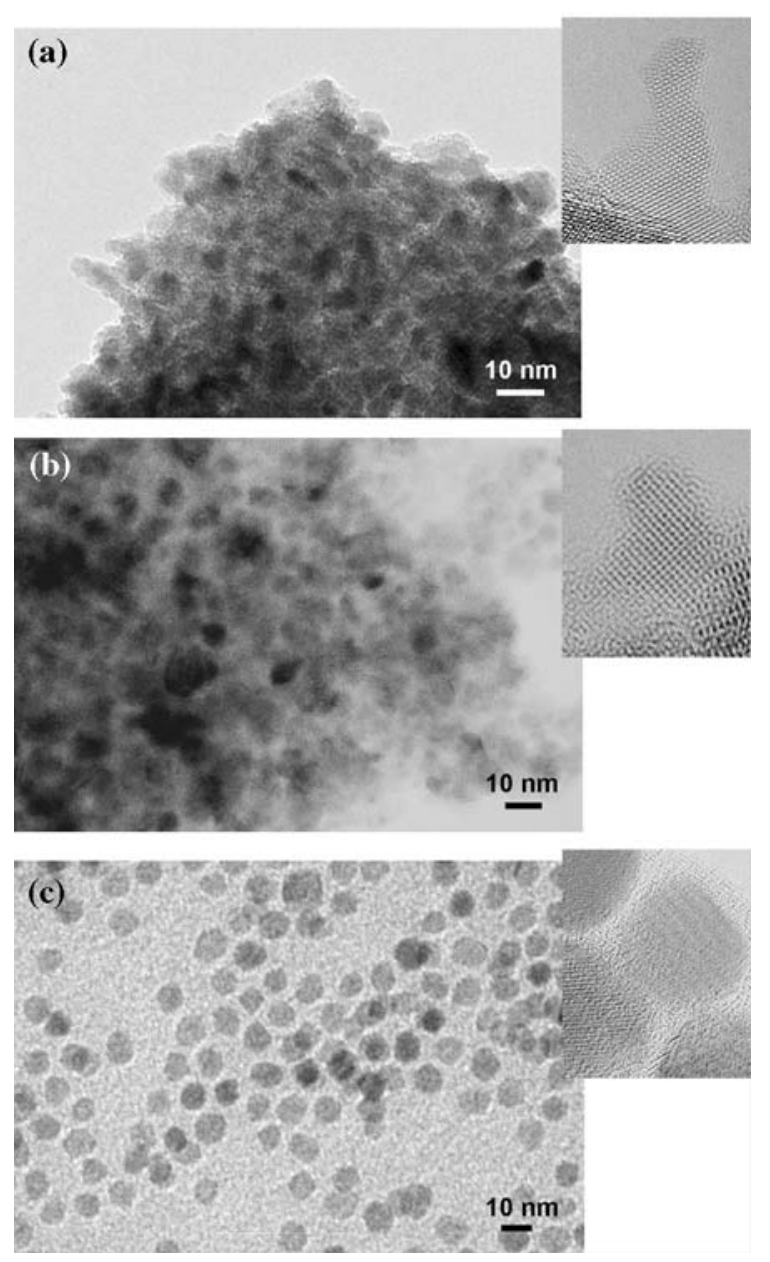

Fig. 2 TEM images of the Co-ferrite nanoparticles CP (a), ME (b), and TD (c) with the corresponding HREM images of the individual nanoparticles dispersion of $11 \%$, visually measured on 500 nanoparticles from BF TEM images).

The nanoparticles prepared by all three methods were well crystalline as evident from HREM images (insets of Fig. 2).

The quantitative EDS analysis showed considerable variations in the composition of the nanoparticles. The standard deviations of the measurements were comparable to the deviation of the method itself (determined on the $\mathrm{CoFe}_{2} \mathrm{O}_{4}$ ceramic standard) indicating homogeneous distribution of cations between the nanoparticles. The nanoparticles prepared by the co-precipitation in microemulsions (sample ME) showed composition with the cobalt-to-iron atomic ratio close to the stoichiometric value of 0.5 (Table 1), whereas for the nanoparticles co-precipitated from aqueous solutions (sample $\mathrm{CP}$ ) the $\mathrm{Co} / \mathrm{Fe}$ ratio was slightly smaller than the stoichiometric. For the nanoparticles prepared by thermal decomposition (sample TD), this value was only half of the stoichiometric (Table 1). In the nanoparticles TD only half of the cobalt atoms added was incorporated into the spinel structure. Due to high $\mathrm{pH}$ value used in both coprecipitation techniques, cobalt was completely precipitated and incorporated into the spinel ferrite. Higher thermal stability of cobalt oleate compared to iron oleate is most likely the reason for the lower content of cobalt incorporated into the spinel structure during the thermal decomposition.

The composition of the nanoparticles $\mathrm{TD}$ is actually outside the spinel range of the $\mathrm{Co}-\mathrm{Fe}-\mathrm{O}$ phase diagram (Pelton et al. 1979). As shown in the 
case of Zn-ferrite (Makovec and Drofenik 2008; Makovec et al. 2009), the spinel nanoparticles can sustain large deviations from their stoichiometry without losing their single-phase nature. When the nanoparticles TD were heated in air to $600{ }^{\circ} \mathrm{C}, \mathrm{Fe}_{2} \mathrm{O}_{3}$ was precipitated, whereas the nanoparticles $\mathrm{CP}$ and ME remained in the single spinel phase after heating.

Figure 3 shows Fe XANES profiles for the synthesized nanoparticles and some standard compounds. The Fe K edge for both co-precipitated nanoparticles $\mathrm{CP}$ and $\mathrm{ME}$ was at the same energy position as in $\mathrm{CoFe}_{2}{ }^{3+} \mathrm{O}_{4}$ reference. For the nanoparticles TD, the edge was shifted for $0.5 \mathrm{eV}$ toward lower energies, and was at the same position as the edge in $\mathrm{Fe}_{3} \mathrm{O}_{4}$ reference. The edge shift is best observed at the edge mid-point indicated by the arrow for $\mathrm{Fe}_{3} \mathrm{O}_{4}$ spectrum in Fig. 3. The edge shift was quantitatively evaluated by shifting and overlapping the compared spectra or their derivatives in the energy region of the edge slope, by a best fit procedure. From the spectra of the reference samples with known iron oxidation states, we have found that the Fe K edge is shifted for about $4 \mathrm{eV}$ per valence unit in agreement with previous observations (Arčon et al. 2007). Based on the

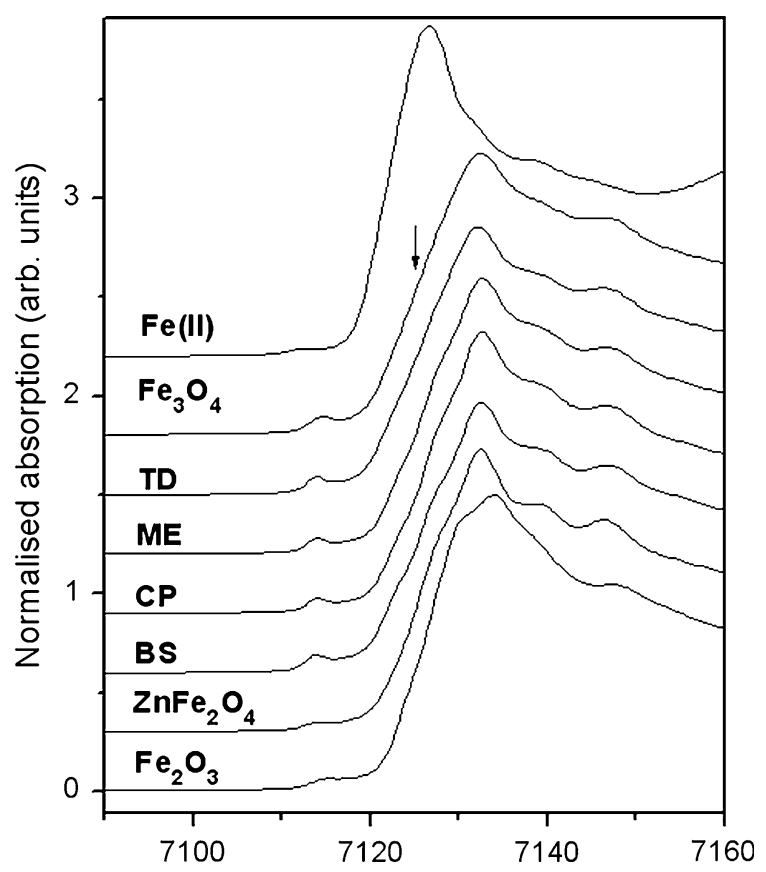

Fig. 3 Fe XANES spectra measured on synthesized nanoparticles and reference compounds $\mathrm{Fe}_{2} \mathrm{O}_{3}, \mathrm{CoFe}_{2} \mathrm{O}_{4}, \mathrm{ZnFe}_{2} \mathrm{O}_{4}$, $\mathrm{Fe}_{3} \mathrm{O}_{4}$, and $\mathrm{LiFePO}_{4}$ as a standard for $\mathrm{Fe}(\mathrm{II})$. Arrow indicates the edge mid-point on the $\mathrm{Fe}_{3} \mathrm{O}_{4}$ spectrum comparison of the edge energy with the reference spectra, we can conclude that the average iron valences are $3+$ in the co-precipitated samples $\mathrm{CP}$ and $\mathrm{ME}$ and 2.7+ in the sample TD. Considering Codeficient composition, the lower iron valence in the sample TD is expected. Presuming that no cation vacancies would form in the structure of the nanoparticles TD, the average $\mathrm{Fe}$ valence can be calculated to be $2.83+\left(\mathrm{Co}_{0.6} \mathrm{Fe}_{0.4}^{2+} \mathrm{Fe}_{2}{ }^{3+} \mathrm{O}_{4}\right)$. The lower iron valence in the sample TD is therefore a consequence of its Co-deficient composition and the slightly reducing thermodynamic conditions during their synthesis defined with the elevated temperature and the environment of the organic medium.

The local structure of the nanoparticles has been studied using EXAFS. In Fig. 4, the Fourier transforms of the EXAFS spectra of the nanoparticles are compared with the spectra of the larger particles BS, which served as standard for the "bulk" Co-ferrite. The quality of the experimental material can be judged from EXAFS signal, $k^{3} \chi(k)$ graphs given in Supplement 2.

Some conclusions on the structure of the nanoparticles can already be made by inspection of the EXAFS spectra. The first peak in the FT EXAFS spectra around $\sim 1.6 \AA$ corresponds to first neighborsoxygen ions comprising the oxygen polyhedra (The corresponding interatomic distance after phase correction is close to $2 \AA$-the same for both metal ions.). The amplitude of the first peak depends somewhat on the site of the absorber-ion incorporation. The incorporation at octahedral sites $(\mathrm{CN}=6)$ will produce higher amplitude of the first peak compared to the incorporation at tetrahedral sites $(\mathrm{CN}=4)$. The scattering from the second neighbors forms the next wide peak in the FT EXAFS spectra, apparently composed of two components or subpeaks centered at $\sim 2.6$ and $\sim 3.1 \AA$ (corresponding to real distances of 3.0 and $3.6 \AA$, respectively). The relative amplitudes of those peaks depend strongly on the site of the absorber cation. The octahedral site has 6 second neighbors at the distance of $3.0 \AA$ and 6 at the distance of $3.5 \AA$. The cation at a tetrahedral site has 12 second neighbors at the distance of $3.5 \AA$ and 4 second neighbors at the distance of $3.6 \AA$. Thus, a subpeak in the FT EXAFS centered at $\sim 2.6 \AA$ (marked in Fig. 4 with $\mathrm{B}$ ) indicates the incorporation of the absorber cation at the octahedral or B site, while the subpeak centered at $3.1 \AA$ (marked in Fig. 4 with A) indicates 


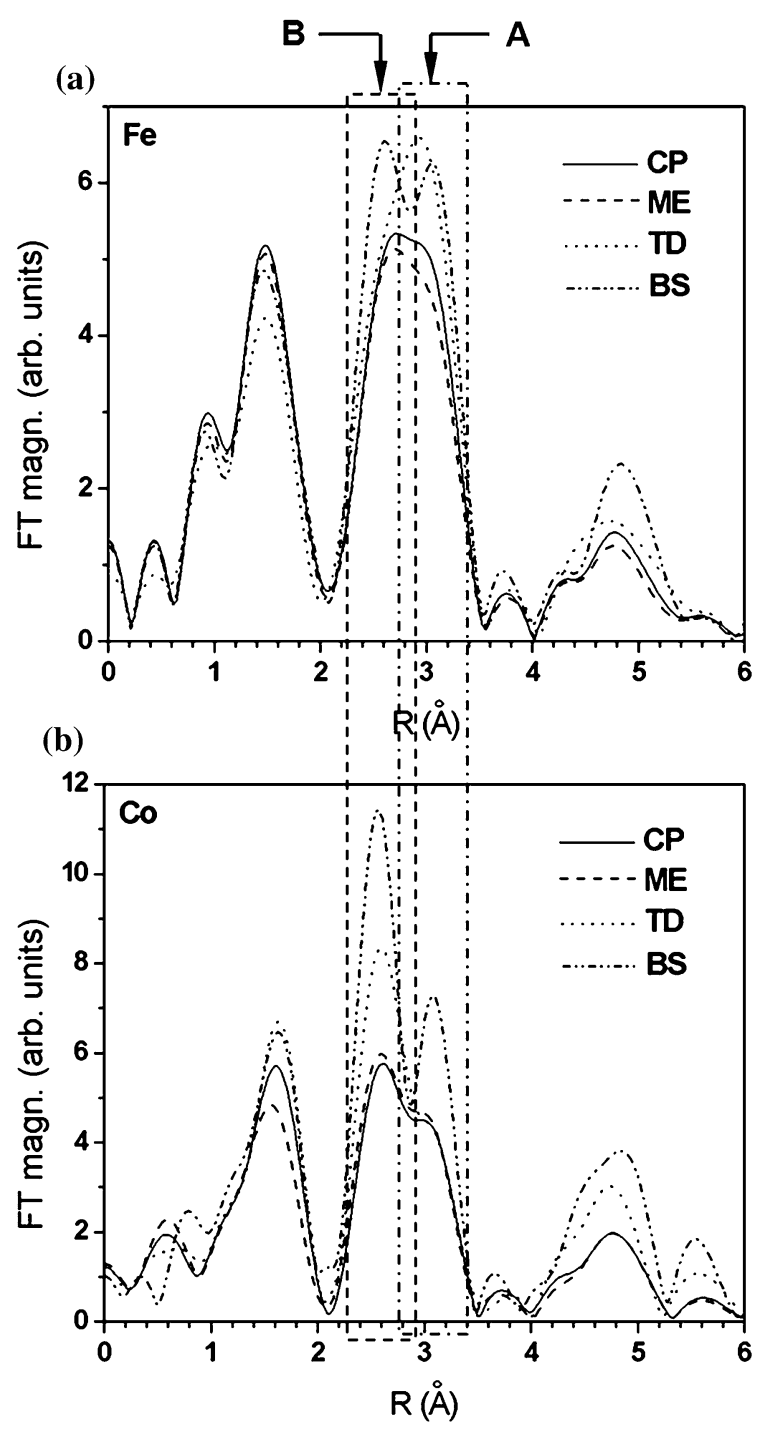

Fig. 4 FT EXAFS k ${ }^{3}$-weighted spectra from Fe (a) and Co (b) $K$ edge for the Co-ferrite nanoparticles CP, ME, and TD

the absorber cation incorporated at a tetrahedral lattice site (A-site).

Comparison between the Co FT EXAFS spectra for the different samples shows that the annealed nanoparticles BS, as well as the sample TD display the larger first peak and dominant subpeak B of the second-neighbor peak, in accordance with the predominant incorporation of cobalt at the octahedral sites. The first peak and the subpeak B are relatively smaller in the co-precipitated nanoparticles $\mathrm{CP}$ and $\mathrm{ME}$ suggesting partial migration of cobalt to tetrahedral sites. As expected, the opposite trend is visible when comparing the Fe spectra.
To give these observations a firm quantitative basis, the EXAFS spectra were modeled with an ab initio FEFF calculation (Ravel and Newville 2005) using a simultaneous fit of the spectra of the two metals as described earlier (Calvin et al. 2002). Four FEFF models, with either metal occupying sites $\mathrm{A}$ and $\mathrm{B}$, were combined, each built on the spinel crystallographic data with lattice constant $a=8.49800 \AA$ and oxygen parameter 0.3866 , comprising the first seven single-scattering paths up to $3.75 \AA$. The four multiple-scattering paths in the range were neglected for low estimated probability $(<6.5 \%)$ and wide spread in the $R$ space. It should be noted that the focusing paths with strong and sensitive forward-scattering amplitude, arise in spinel only from $a / 2=4.25 \AA$ on.

The included paths are, in addition to the intrinsic EXAFS parameters $E_{0}$ and $S_{0}^{2}$ for each metal, described by the standard parameters, the DebyeWaller widths, separately for the site types (A-A, $\mathrm{A}-\mathrm{B}$, and $\mathrm{B}-\mathrm{B})$. The $\mathrm{Fe}-\mathrm{O}$ and $\mathrm{Co}-\mathrm{O}$ distances are treated separately, other distances are taken from the crystallographic model and described by only two parameters, the general linear expansion coefficient and the spinel-specific oxygen parameter. The site occupation probabilities for $\mathrm{Fe}$ and $\mathrm{Co}$ are constrained to unit sums.

Figure 5 shows the results of least-square relaxation of the EXAFS spectra of the sample TD to the model for the $R$-range 1.1-3.5 $\AA$, using $k^{3}$ weight in the $k$-range $3.6-11.5 \AA^{-1}$, for $\mathrm{Fe}$ spectra and 3.6$12.5 \AA^{-1}$ for Co spectra. The quality of the fits ( $r$-factor) is satisfactory, always better then $1.4 \%$. The complete results of the least-square relaxation of the EXAFS spectra to the model can be found in Supplement 3. The main result of this structural analysis is the degree of inversion $X$ (listed in Table 1), showing migration of Co from octahedral sites typical for $\mathrm{CoFe}_{2} \mathrm{O}_{4}$ bulk to tetrahedral sites in the nanoparticles. In the $\mathrm{Co}-$ ferrite particles $\mathrm{BS}$ prepared by annealing at $600{ }^{\circ} \mathrm{C}$, which were considered as the bulk standard, cobalt is indeed situated almost completely at the octahedral lattice sites $(X=0.931)$. Similarly to bulk, cobalt retained predominantly the octahedral sites also in the nanoparticles TD synthesized using thermal decomposition ( $X=0.926)$. However, for the co-precipitated nanoparticles $\mathrm{CP}$ and $\mathrm{ME}$ the degree of inversion was estimated to be considerably smaller then the bulk value $(X \sim 0.75)$. 
Fig. 5 FT magnitude of the $\mathrm{k}^{3}$-weighted $\mathrm{Fe}$ (a) and Co (b) EXAFS data (solid line) with the model (dashed) for the $\mathrm{Co}-$ ferrite nanoparticles TD

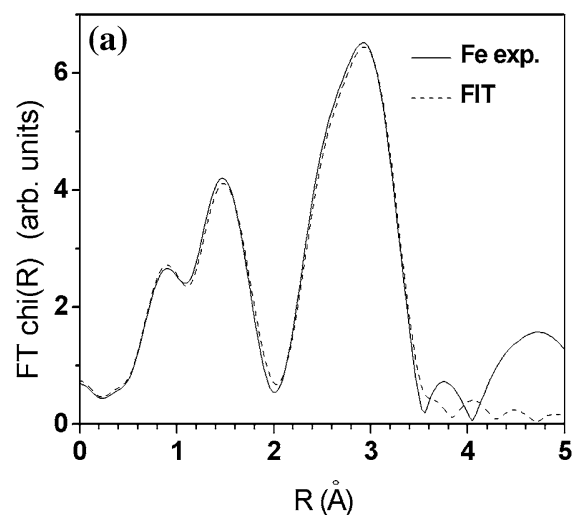

In agreement with differences observed in composition and structural properties, the nanoparticles also show markedly different magnetic properties. The room-temperature magnetization curves of the nanoparticles suggests superparamagnetic behavior (Fig. 6), showing zero coercitivity.

Nevertheless, the magnetization measured under zero-field cooling conditions is continuously increasing till $300 \mathrm{~K}$ (Supplement 4) for the nanoparticles $\mathrm{CP}$ and $\mathrm{ME}$, indicating their ferrimagnetic nature. For nanoparticles TD, a broad maximum of zero-field curve exists at temperature $\approx 240 \mathrm{~K}$, revealing their superparamagnetic nature with characteristic blocking temperature $T_{\mathrm{B}}$ around $240 \mathrm{~K}$. The reason for room-temperature superparamagnetic behavior of the nanoparticles TD is most probably related to their dispersed state, where each individual particle is isolated, whereas the co-precipitated nanoparticles $\mathrm{CP}$ and ME of comparable size are agglomerated and consequently ferrimagnetic due to interparticle magnetic interactions. Even at relatively high fields, the saturation magnetization of the nanoparticles was not

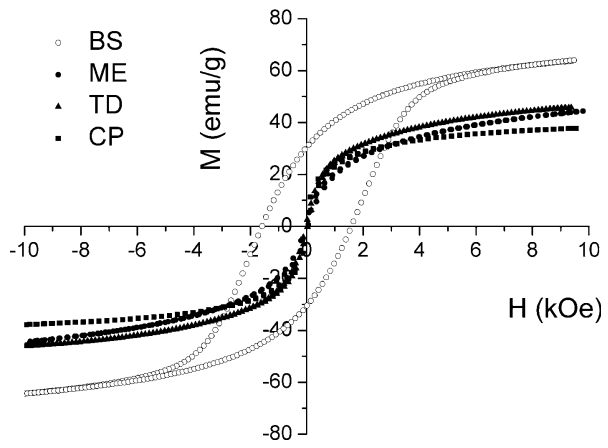

Fig. 6 Room-temperature magnetization curves for the $\mathrm{Co}-$ ferrite nanoparticles $\mathrm{CP}, \mathrm{ME}$, and TD and standard BS reached, in agreement with the existence of a magnetically inactive layer at their surfaces (Kodama 1999). The room-temperature magnetization curve of the larger Co-ferrite particles BS displays a typical ferrimagnetic behavior with large coercivity and saturation magnetization in the range found for bulk Co-ferrite (Smit and Wijn 1959) (Fig. 6).

Structural details, obtained from EXAFS experiments, are key factors for explaining low temperature magnetization measurements. These provide, at temperatures close to $0 \mathrm{~K}$ the information about distribution of cations within the spinel lattice. Namely, at $0 \mathrm{~K}$, saturation magnetization directly reflects the difference in magnetization of the tetrahedral and the octahedral sublattices. Magnetization curves, measured at $10 \mathrm{~K}$, all display typical ferrimagnetic response (Fig. 7).

The nanoparticles samples reach a lower saturation magnetization value than calculated for the known distribution of cations, in agreement with the existence of magnetically dead surface layer (Kodama 1999). On the contrary, the larger Co-ferrite particles BS

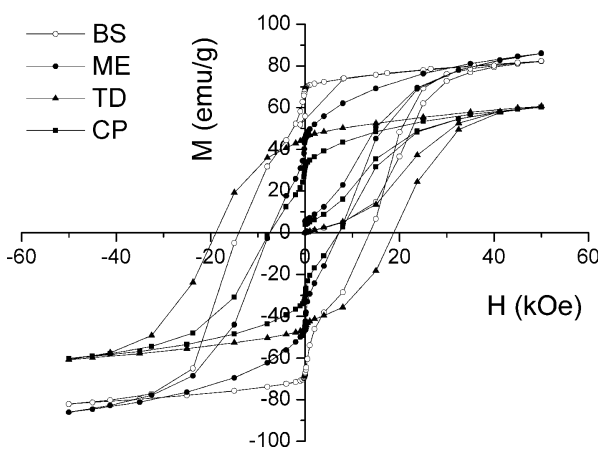

Fig. 7 Hysteresis loops for the Co-ferrite nanoparticles $\mathrm{CP}$, $\mathrm{ME}$, and TD and standard BS measured at $10 \mathrm{~K}$ 
reach saturation magnetization typical for the bulk Co-ferrite (Smit and Wijn 1959). Due to its lowest degree of inversion, the nanoparticles ME exhibit the largest value of saturation magnetization (magnetic moment per molecule of $\mathrm{CoFe}_{2} \mathrm{O}_{4}$ increases from $3 \mathrm{Bohr}$ magnetons for inverse spinel to $7 \mathrm{Bohr}$ magnetons for normal spinel). For the nanoparticles $\mathrm{CP}$, one would expect higher saturation magnetization value, since they display just slightly higher degree of inversion to that of the nanoparticles ME. The reason for relatively low magnetization of the $\mathrm{CP}$ nanoparticles is most probably related to the broad size distribution and the presence of very small nanoparticles that reduce sample's saturation magnetization. Sample TD exhibits the lowest saturation magnetization in agreement with its almost completely inverse structure.

Due to their composition close to that of the stoichiometric $\mathrm{CoFe}_{2} \mathrm{O}_{4}$, the largest coercivity values would be expected for the co-precipitated nanoparticles CP and ME. However, the presence of cobalt ions at the tetrahedral sublattice sites strongly reduces the coercivity (Franco and Zapf 2008; SalazarAlvarez et al. 2007). In spite of the substantial lack of cobalt in the composition of the nanoparticles TD, they exhibit the largest value of coercivity and the $M_{\mathrm{r}} / M_{\mathrm{S}}$ ratio close to the value expected for isolated particles with cubic anisotropy due to their inverse spinel structure. The difference between coercivity of the co-precipitated nanoparticles and the nanoparticles synthesized by the thermal decomposition illustrates the large importance of the cation distribution. The larger Co-ferrite particles BS display the $M_{\mathrm{r}} / M_{\mathrm{S}}$ ratio of 0.84 reaching the value typical for the bulk $\mathrm{CoFe}_{2} \mathrm{O}_{4}$ (0.83), but they display a lower value of coercivity than the magnetically hardest nanoparticles TD. The reason for the higher coercivity in the smaller nanoparticles is most probably related to the surface effect. The fraction of the surface atoms with broken symmetry is much larger in the smaller nanoparticles resulting in an increase in the surface anisotropy, thus increasing the effective magnetic anisotropy and consequently the coercivity (Kodama 1999).

The reason for the different cation distributions in the samples prepared by different methods is in unique thermodynamic environment during their synthesis and in the flexibility of the structure characteristic for spinel nanoparticles. The major difference in the presented methods is the temperature of synthesis. Co-precipitations (in water or microemulsions) are low-temperature processes. At low temperatures the equilibrium structure is not easily formed. On the contrast, the thermal decomposition was performed at an elevated temperature of $290{ }^{\circ} \mathrm{C}$, where a near-equilibrium structure was formed despite the small size of the nanoparticles.

\section{Conclusions}

Structural and magnetic properties of Co-ferrite nanoparticles having uniform particle size around $8 \mathrm{~nm}$ synthesized using three different methods were compared. A simple co-precipitation from aqueous solutions and a co-precipitation in an environment of microemulsions are low temperature methods $\left(50{ }^{\circ} \mathrm{C}\right)$, whereas a thermal decomposition of organo-metallic complexes was performed at elevated temperature of $290{ }^{\circ} \mathrm{C}$. The nanoparticles synthesized with thermal decomposition showed a Co-deficient composition $\left(\mathrm{Co}_{0.6} \mathrm{Fe}_{2.4} \mathrm{O}_{4}\right)$, the average Fe valence of $2.7+$, and almost completely inverse spinel structure, similar to that of the bulk. Both co-precipitated samples were nearly stoichiometric, had Fe valence of $3+$, yet they showed a significant proportion of cobalt ions incorporated at the tetrahedral sites of their spinel structure. Magnetic properties of the nanoparticles were in good agreement with their composition and the cation distribution in their structure.

Acknowledgments This work was supported by the Slovenian Research Agency, the Ministry of Higher Education, Science and Technology of the Republic of Slovenia within the National Research Program, and by DESY and the European Community under Contract RII3-CT-2004-506008 (IA-SFS). Provision of synchrotron radiation facilities by HASYLAB is acknowledged. The authors would also like to thank Daša Lesjak for help with the synthesis of the nanoparticles, E. Welter of HASYLAB for expert advice on beamline operation and Paul McGuiness for performing VSM measurements.

\section{References}

Ammar S, Jouini N, Fievet F, Stephan O, Marhic C, Richard M, Villain F, Chartier dit Moulin C, Brice S, Sainctavit P (2004) Influence of the synthesis parameters on the cation distribution of $\mathrm{ZnFe} 2 \mathrm{O} 4$ nanoparticles obtained by forced hydrolysis in polyol medium. J Non-Cryst Solids 345\&346: 658-662 
Arčon I, Kolar J, Kodre A, Hanžel D, Strlič M (2007) XANES analysis of $\mathrm{Fe}$ valence in iron gall inks. X-ray spectrom 36:199-205

Arruebo M, Fernández-Pacheco R, Ibarra MR, Santamaría J (2007) Magnetic nanoparticles for drug delivery. Nano Today 2:22-32

Batlle X, Labarta A (2002) Finite-size effect in fine particles: magnetic and transport properties. J Phys D 35:R15-R42

Calvin S, Carpenter EE, Ravel B, Harris VG, Morrison SA (2002) Multiedge refinement of extended X-ray absorption fine structure of manganese zinc ferrite nanoparticles. Phys Rev B66:224405-1-224405-13

Carpenter EE, O'Connor CJ, Harris VG (1999) Atomic structure and magnetic properties of $\mathrm{MnFe}_{2} \mathrm{O}_{4}$ nanoparticles produced by reverse micelle synthesis. J Appl Phys 85: $5175-5177$

Cullity BD (1987) Elements of X-ray diffraction. AddisonWesley, Reading

Elster A, Burdette J (2001) Questions and answers in magnetic resonance imaging. Mosby, St. Louis

Fontjin WFJ, van der Zaag PJ, Feiner LF, Metselaar R, Devillers MAC (1999) A consistent interpretation of the magneto-optical spectra of spinel type ferrites (invited). J Appl Phys 85:5100-5105

Franco A, Zapf V (2008) Temperature dependence of magnetic anisotropy in nanoparticles of $\mathrm{Co}_{x} \mathrm{Fe}_{(3-x)} \mathrm{O}_{4}$. J Magn Magn Mater 320:709-713

Gilchrist RK, Medal R, Shorey WD, Hanselman RC, Parrott JC, Taylor CB (1957) Selective inductive heating of lymph nodes. Ann Surg 146:596-606

Häfeli U, Schüt W, Teller J, Zborowski M (1997) Scientific and clinical applications of magnetic carriers. Plenum, New York

Hamdeh HH, Ho JC, Oliver SA, Willey RJ, Oliveri G, Busca GJ (1997) Magnetic properties of partially-inverted zinc ferrite aerogel powders. J Appl Phys 81:1851-1857

Jeyadevan B, Tohji T, Nakatsuka KJ (1994) Structure-analysis of coprecipitated $\mathrm{ZnFe}_{2} \mathrm{O}_{4}$ by extended X-ray absorption fine-structure. Appl Phys 76:6325-6327

Kamiyama T, Haneda K, Sato T, Ikeda S, Asano H (1992) Cation distribution in $\mathrm{ZnFe}_{2} \mathrm{O}_{4}$ fine particles studied by neutron powder diffraction. Solid State Commun 81:563-566

Kodama RH (1999) Magnetic nanoparticles. J Magn Magn Mater 200:359-372

Li S, John VT, O'Connor, Harris VG, Carpenter E (2000) Cobalt ferrite nanoparticles: Structure, cation distributions and magnetic properties. J Appl Phys 87:6223-6225

Makovec D, Drofenik M (2008) Non-stoichiometric zinc-ferrite spinel nanoparticles. J Nanopart Res 10:131-141

Makovec D, Košak A, Drofenik M (2004) The preparation of $\mathrm{MnZn}$-ferrite nanoparticles in water-CTAB-hexanol microemulsions. Nanotechnology 15:S160-S166

Makovec D, Kodre A, Arčon I, Drofenik M (2009) Structure of manganese zinc ferrite spinel nanoparticles prepared with co-precipitation in reversed microemulsions. J Nanopart Res 11:1145-1158
Morrison SA, Cahill CL, Carpenter EE, Calvin S, Swaminathan R, McHenry ME, Harris VG (2004) Magnetic and structural properties of nickel zinc ferrite nanoparticles synthesized at room temperature. J Appl Phys 95:63926395

Mosbach K, Schröder U (1979) Preparation and application of magnetic polymers for targeting of drugs. FEBS Lett 102:112-116

Pankhurst QA, Conolly J, Jones SK, Dobson J (2003) Applications of magnetic nanoparticles in biomedicine. J Phys D 36:R167-R181

Park J, An K, Hwang Y, Park JG, Noh HJ, Kim JY, Park JH, Hwang NM, Hyeon T (2004) Ultra-large-scale synthesis of monodispersed nanocrystals. Nature Mater 3:891-895

Pelton AD, Schmalzried H, Sticher J (1979) Thermodynamics of Mn3O4-Co3O4, Fe3O4-Mn3O4, and Fe3O4-Co3O4 spinels by phase-diagram analysis. Ber Bunsen-Ges Phys Chem 83:241-252

Pileni MP (1993) Reverse miceles as microreactors. J Phys Chem 97:6961-6973

Ravel B, Newville M (2005) ATENA, ARTHEMIS, HEPHAESTUS: data analysis for X-ray absorption spectroscopy using IFEFFIT. J Synchrotron Radiat 12:537-541

Rosenweig R (1932) Ferrohydrodynamics. Cambridge University Press, Cambridge

Salazar-Alvarez G, Olsson RT, Sort J, Macedo AA, Ardisson JD, Baró MD, Gedde UW, Nogués J (2007) Enhanced coercivity in Co-rich near-stoichiometric $\mathrm{Co}_{x} \mathrm{Fe}_{3-x} \mathrm{O}_{4+\delta}$ nanoparticles prepared in large batches. Chem Mater 19:4957-4963

Sato T, Haneda K, Seki M, Iijima T (1990) Morphology and magnetic properties of ultrafine $\mathrm{ZnFe}_{2} \mathrm{O}_{4}$ particles. Appl Phys A50:13-16

Senyei A, Widder K, Czerlinski C (1978) Magnetic guidance of drug-carrying microspheres. J Appl Phys 49:3578-3583

Sivakumar N, Narayanasamy A, Shinoda K, Chinnasamy CN, Jeyadevan B, Greneche JM (2007) Electrical and magnetic properties of chemically derived nanocrystalline cobalt ferrite. J Appl Phys 102:013916 1-8

Smit J, Wijn HPJ (1959) Ferrites. Philips' Technical Library, Eindhoven, The Netherlands

Sugimoto T (2001) Monodispersed particles. Elsevier science, Amsterdam

Sun S, Zeng H, Robinson DB, Raux S, Rice PM, Wang SX, Li $\mathrm{G}$ (2004) Monodisperse $\mathrm{MFe}_{2} \mathrm{O}_{4}(\mathrm{M}=\mathrm{Fe}, \mathrm{Co}, \mathrm{Mn})$ nanoparticles. J Am Chem Soc 126:273-279

Tirosh E, Shemer G, Markovich G (2006) Optimizing cobalt ferrite nanocrystal synthesis using a magneto-optical probe. Chem Mater 18:465-470

Veverka M, Veverka P, Kaman O, Lancok A, Zaveta K, Pollert E, Knizek K, Bohacek J, Benes M, Kaspar P, Duguet E, Vasseur S (2007) Magnetic heating by cobalt ferrite nanoparticles. Nanotechnology 18:345704 1-7

Widder KJ, Senyei AE, Scrapelli DG (1978) Magnetic microspheres: a model system for site specific drug delivery in vivo. Proc Soc Bio Exp Biol Med 58:141-146 